Cite this: Phys. Chem. Chem. Phys., 2011, 13, 10318-10325

\title{
Effect of ionic strength and type of ions on the structure of water swollen polyelectrolyte multilayers $\dagger \ddagger$
}

\author{
S. Dodoo, ${ }^{a}$ R. Steitz, ${ }^{b}$ A. Laschewsky ${ }^{c}$ and R. von Klitzing ${ }^{* a}$ \\ Received 28th July 2010, Accepted 31st March 2011 \\ DOI: $10.1039 / \mathrm{c0cp01357a}$
}

This study addresses the effect of ionic strength and type of ions on the structure and water content of polyelectrolyte multilayers. Polyelectrolyte multilayers of poly(sodium-4-styrene sulfonate) (PSS) and poly(diallyl dimethyl ammonium chloride) (PDADMAC) prepared at different $\mathrm{NaF}, \mathrm{NaCl}$ and $\mathrm{NaBr}$ concentrations have been investigated by neutron reflectometry against vacuum, $\mathrm{H}_{2} \mathrm{O}$ and $\mathrm{D}_{2} \mathrm{O}$. Both thickness and water content of the multilayers increase with increasing ionic strength and increasing ion size. Two types of water were identified, "void water" which fills the voids of the multilayers and does not contribute to swelling but to a change in scattering length density and "swelling water" which directly contributes to swelling of the multilayers. The amount of void water decreases with increasing salt concentration and anion radius while the amount of swelling water increases with salt concentration and anion radius. This is interpreted as a denser structure in the dry state and larger ability to swell in water (sponge) for multilayers prepared from high ionic strengths and/or salt solution of large anions. No exchange of hydration water or replacement of $\mathrm{H}$ by D was detected even after eight hours incubation time in water of opposing isotopic composition.

\section{Introduction}

Thin polymer films are widely used for modification and functionalization of surfaces. The adsorption from solution is a particularly refined technology for modifying surfaces for advanced applications. It is well documented that the physisorption or chemisorption of polyelectrolytes or reactive polymers onto surface-functionalized substrates can lead to the deposition of molecularly thin surface films. ${ }^{1-6}$

Reflectivity techniques, especially neutron and X-ray reflectometry, are well suited for the characterization of multilayer films, as they allow the determination of the concentration gradient along the layer normal. X-Ray reflectometry has only exhibited Kiessig fringes that arise from the interference of X-ray beams reflected at the substrate-film and film-air interfaces. $^{7-9}$ Neutron reflectivity measurements of polymer

${ }^{a}$ Technische Universität Berlin, Stranski-Laboratorium, Department of Chemistry, TU Berlin, Strasse des 17. Juni 124, D-10623 Berlin, Germany.E-mail: klitzing@chem.tu-berlin.de;

Fax: + 49 (0)30 31426602; Tel: + 49 (0)30 31423931

${ }^{b}$ Helmholtz-Zentrum Berlin für Materialien und Energie GmbH,

Hahn-Meitner-Platz 1, D-14109 Berlin, Germany

${ }^{c}$ Institut für Chemie, Universität Potsdam, Karl-Liebknecht-Str. 24-25, D-14476 Potsdam-Golm, Germany

$\dagger$ Electronic supplementary information (ESI) available: See DOI: $10.1039 / \mathrm{c} 0 \mathrm{cp} 01357 \mathrm{a}$

$\ddagger$ This article was submitted as part of a Themed Issue on Scattering Methods Applied to Soft Matter. Other papers on this topic can be found in issue 8 of vol. 13 (2011). This issue can be found from the $P C C P$ homepage [http://www.rsc.org/pccp] films of a superlattice structure showed that the polyelectrolytes are deposited as layers with an interdigitation smaller or equal to a single layer thickness and indicate that there is no distinct layer-by-layer separation between polyelectrolytes of opposite charges. ${ }^{10-12}$ The main driving force of film assembly is entropy due to release of counterions. Measurements of the surface potential resulted in a change of (surface) potential after each adsorption step, i.e. after each additional single deposited polyelectrolyte layer. $^{13-16}$

For alternating layers of poly(styrene sulfonate), PSS, and poly(diallyl dimethyl ammonium chloride), PDADMAC, adsorbed onto smooth surfaces, a roughening of successively deposited layers leads to a progressively larger number of adsorption sites for consecutive generations of adsorbed polymers, and thus to an increase in layer thicknes with increasing number of deposited layers. The thickness of the deposited layer may be fine-tuned via the ionic strength of the solutions used for preparation. ${ }^{17}$

The difference in film thickness is explained by different conformations of the chains: without salt the polyelectrolyte chains are oriented flat and parallel to the substrate, with higher salt concentrations of the aqueous solutions the chains form coils ${ }^{18,19}$ which are then adsorbed at the interface. Because of screening of charges along the polyelectrolyte chains the polymer is more entangled with larger thickness. TIRF (Total Internal Reflection Fluorescence) kinetic experiments, where the fluorescence of a fluorescein labeled polyelectrolyte is measured, indicate an increasing amount of 
adsorbate with increasing salt concentration. Measurements of the rhodamine transport through the polyelectrolyte multilayer reveal a higher diffusion coefficient of rhodamine for multilayers prepared without salt. These findings lead to the conclusion that the polyelectrolyte density of multilayers prepared without salt is lower than the polyelectrolyte density of multilayers prepared with salt additive. ${ }^{20}$ The type of salt $^{21-24}$ used in the multilayer preparation influences the thickness of the multilayer according to the Hofmeister effect.

Due to the high solubility of polyelectrolytes in water polyelectrolyte multilayers are very sensitive to the water content of the environment. This is of interest since polyelectrolyte multilayers are often used as cushions or junctions between different materials. Further, they present building blocks for novel stimuli responsive materials. Therefore, it is necessary to conduct structural investigations for clarifying their internal structure. Swelling behaviour of polyelectrolyte multilayers has been investigated in recent times by ellipsometry, X-ray and neutron reflectometry. Wong et al. ${ }^{25}$ observed an "odd-even effect" which shows outer-layer dependence for a poly(sodium-4-styrene sulfonate) (PSS)/poly(allylamine hydrochloride) (PAH) multilayer in ellipsometric swelling experiments in $99 \%$ relative humidity. Ellipsometrically determined swelling percentages of PSS/PDADMAC multilayers prepared in $0.5 \mathrm{M}$ $\mathrm{NaCl}$ show that water uptake can vary depending on whether the capping layer is a polycation or a polyanion. ${ }^{26}$ Steitz et al. ${ }^{27}$ reported that PSS/PAH systems swell as much as $56 \%$ in the outer layer and $42 \%$ in the inner part by neutron reflectometry, where two boxes of different scattering length densities were needed to fit the reflectometry curves. PSS/PAH bilayers studied by X-ray and neutron reflectometry as a function of the type of salt shows that the PSS/PAH bilayer thickness is independent of the kind of salt $(\mathrm{NaCl}$ or $\mathrm{KCl})$, yet its composition is different (more bound water for $\mathrm{NaCl}){ }^{28}$

Especially in biologically relevant systems like in the presence of proteins, specific ion effects become important. So far, a detailed study on specific anion effects on the water content and the inner structure of polyelectrolyte multilayers is missing. The problem in many studies is that the water content calculated by changes in scattering length density differs from the one calculated by the swelling ratio of multilayer thickness. Here we report on the structural investigation of PSS/PDADMAC multilayers by neutron reflectivity. In order to determine the effect of type of salt and ionic strength on the water content and swelling behaviour of PSS/PDADMAC multilayers, neutron reflectometry experiments were conducted at the solid-liquid and solid-vacuum interfaces.

\section{Experimental section}

\section{Materials and multilayer preparation}

Linear PDADMAC was synthesized by free-radical polymerization of positively charged diallyldimethylammonium chloride (DADMAC) monomers. Details about the synthesis and the characterization are described elsewhere. ${ }^{29,30}$ The molecular weight of the fully charged PDADMAC is $135000 \mathrm{~g} \mathrm{~mol}^{-1}$ and a polydispersity index (PDI) of 1.75. Poly(sodium-4-styrene sulfonate) (PSS) and branched poly(ethylene imine) (PEI) were obtained from Aldrich (Steinheim, Germany). The molecular weight of PEI is $750000 \mathrm{~g} \mathrm{~mol}^{-1}$ and $70000 \mathrm{~g} \mathrm{~mol}^{-1}$ in the case of PSS. PEI and PSS were used as purchased. $\mathrm{NaF}, \mathrm{NaCl}$, and $\mathrm{NaBr}$ were purchased from Merck. Silicon blocks were supplied by Silizium Bearbeitung A. Holm, Tann, Germany, and cleaned for 30 min in piranha solution $\left(\mathrm{H}_{2} \mathrm{O}_{2}: \mathrm{H}_{2} \mathrm{SO}_{4} ; 1: 1\right)$.

The polyelectrolyte multilayers were deposited on the silicon blocks by immersion for $20 \mathrm{~min}$ into aqueous solutions containing $10^{-2}$ mono mol $1^{-1}$ (concentration of monomer units) of the respective polyelectrolytes in $\mathrm{H}_{2} \mathrm{O}$ and by rinsing three times with Milli-Q-water for $1 \mathrm{~min}$ after each deposition step. First, the wafer was immersed in aqueous solution of PEI for $30 \mathrm{~min}$ and then rinsed gently in pure water. This extra step was found to be efficient in the reduction of substrate influence on the adsorption of the next polyelectrolyte layers. ${ }^{31}$ After that PSS and PDADMAC were deposited consecutively via the self-assembly technique. The multilayers were dried in a gentle stream of nitrogen for 3 min after completion of the multilayer assembly. The polyelectrolyte multilayers fabricated were $\mathrm{Si} /$ $\mathrm{PEI} /(\mathrm{PSS} / \mathrm{PDADMAC} 100 \%)_{6}$ at different $\mathrm{NaF}, \mathrm{NaCl}$ and $\mathrm{NaBr}$ concentrations.

\section{Apparatus and measurement procedure}

The homemade sample cell for neutron reflectivity studies of solid/liquid interfaces represents a modified version of the design by Satija and co-workers. ${ }^{32}$ It consists of a poly(tetrafluoroethylene) trough of inner dimensions with $(72 \times 42 \times 3) \mathrm{mm}^{3}$ stainless steel inlet and outlet tubes for the fluid mounted in opposite corners of the trough. It was sealed with a Viton O-ring against the silicon block. A more detailed description of the sample cell is given elsewhere. ${ }^{33}$ All measurements were conducted at room temperature. Neutron reflectivity measurements were performed at the V6 instrument at the HZB Berlin. A detailed description of the instrument can be found in the literature. ${ }^{34}$ A neutron wavelength $=4.66 \AA$ was selected by a graphite monochromator in the incident white beam. The resolution was set by a slit system on the incident side to $\Delta Q=$ $0.001 \AA^{-1}$ for $Q \leq 0.0518 \AA^{-1}$, and $\Delta Q=0.002 \AA^{-1}$ otherwise. A beam of rectangular cross section $0.5 \times 40 \mathrm{~mm}^{2}$ for $Q \leq$ $0.0518 \AA^{-1}$ and $1 \times 40 \mathrm{~mm}^{2}$ for $Q>0.0518 \AA^{-1}$ impinged on the samples at the solid/vacuum interface through vacuum and at the solid/liquid interface through the silicon block.

The scattered neutrons were recorded with a ${ }^{3} \mathrm{He}$-detector in single $\theta / 2 \theta$ steps with a complete run from 0.0047 to $0.1646 \AA^{-1}$ taking typically 6-9 h. Every run was repeated in the low $Q$-range to check for changes in the structure during this time. The off-specular signal was collected simultaneously in a ${ }^{3} \mathrm{He}$ counter offset from the specular position by $0.44^{\circ}$ toward larger angle $2 \theta$. The extinction coefficient of the silicon blocks was found to be $\kappa=0.0038 \mathrm{~mm}^{-1}$ for the monochromatic neutrons used in this work. In the neutron reflectivity experiment at the V6 instrument, the background to the measured intensity was determined to be $(1.5 \pm 0.5) \times 10^{-5}$ for measurements against both liquid phases and $(8 \pm 1) \times 10^{-6}$ for measurements against vacuum. The intensity was normalized on the measured incident intensity $I_{0}$ to obtain the reflectivity $R(Q)$ of the interface. The centerpiece of the experimental setup is shown elsewhere. ${ }^{27}$ A complete measurement cycle 
consisted of four steps: recording neutron reflectivity from the multilayer (1) against vacuum (pressure: from $(0.2-2.7) \times$ $10^{-2}$ mbar) after preparation i.e. after exposure to $\mathrm{H}_{2} \mathrm{O}\left(\mathrm{H}_{2} \mathrm{O}\right.$ vac), (2) against $\mathrm{H}_{2} \mathrm{O}\left(\mathrm{H}_{2} \mathrm{O}\right.$ liq), (3) against $\mathrm{D}_{2} \mathrm{O}\left(\mathrm{D}_{2} \mathrm{O}\right.$ liq) and (4) against vacuum after exposure to $\mathrm{D}_{2} \mathrm{O}\left(\mathrm{D}_{2} \mathrm{O}\right.$ vac).

\section{Fitting procedure}

The neutron reflectivity data were fitted with Parratt's dynamic approach $^{35}$ using the Parratt32 fitting software (provided by HZB). Since all the fabricated multilayers are assumed to have a homogeneous density a so-called one-box model was used to fit the experimental data. Thereby, the multilayer is described by a certain thickness, scattering length density (SLD) and roughness towards the outer medium (vacuum or water). A $\mathrm{SiO}_{2}$ layer with thickness between 5 and $25 \AA$ and with SLD of $3.475 \times 10^{-6} \AA^{-2}$ representing the native oxide layer was established between the Si substrate and PEM. The roughness of the $\mathrm{SiO}_{2}$-interlayer towards silicon and towards the polymer multilayer was kept at zero. The SLD for Si was fixed at $2.073 \times 10^{-6} \AA^{-2}$ throughout the fitting. First, the $\mathrm{H}_{2} \mathrm{O}$ vac data were fitted. Then, the best fit for the $\mathrm{SiO}_{2}$ thickness was kept fixed for a particular substrate for all other conditions, i.e. $\mathrm{H}_{2} \mathrm{O}$ liq, $\mathrm{D}_{2} \mathrm{O}$ liq and $\mathrm{D}_{2} \mathrm{O}$ vac. This fitting procedure was applied to all the polyelectrolyte multilayers except for the multilayer prepared from $0.25 \mathrm{M} \mathrm{NaCl}$. In this case, the $\mathrm{SiO}_{2}$ layer went to thickness values smaller than $5 \AA$, which is physically senseless. For the very reason the $\mathrm{SiO}_{2}$ layer was omitted from the model and replaced by a roughness term instead. All previously fitted samples were cross-checked with this second fitting approach and no significant differences were observed in the derived parameters.

\section{Results}

\section{Hydration water in a dry polyelectrolyte multilayer}

Fig. 1 shows the raw neutron reflectivity data (symbols) of PSS/ PDADMAC sample and the best fit (solid line). The spectra contain Kiessig oscillations but no Bragg peaks suggesting that

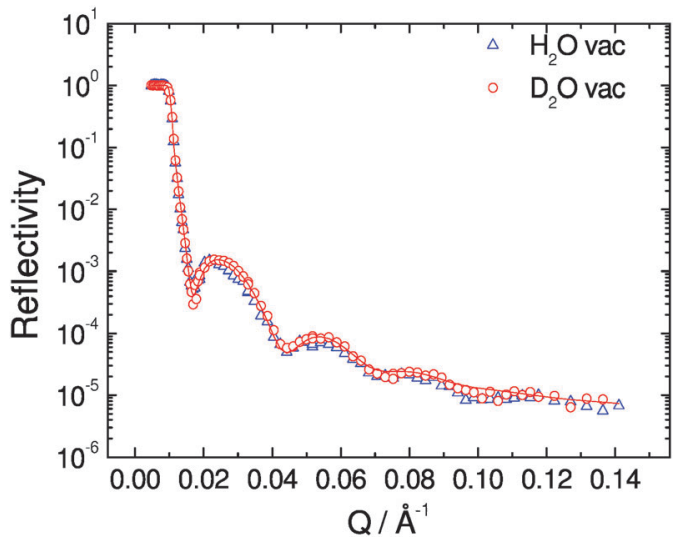

Fig. 1 Neutron reflectivity against vacuum of (PSS/PDADMAC) 6 prepared in $0.1 \mathrm{M} \mathrm{NaBr}$ aqueous solution and measured against vacuum after exposure to $\mathrm{H}_{2} \mathrm{O}$ and $\mathrm{D}_{2} \mathrm{O}$, respectively. The data points are supplemented with a continuous line representing the best fit model as described in the fitting procedure. there is no pronounced density variation within the repeat units of the multilayer.

The sample (PSS/PDADMAC) 6 prepared from aqueous solution of $0.1 \mathrm{M} \mathrm{NaBr}$ was measured against vacuum. The Kiessig oscillations of the multilayer against $\mathrm{H}_{2} \mathrm{O}$ vac and $\mathrm{D}_{2} \mathrm{O}$ vac coincide i.e., the oscillation amplitudes and the minima positions are the same, which means equal thickness, SLD and roughness of the multilayer. The best fit of the experimental data results in a thickness of $229 \pm 5 \AA$, SLD of $(1.04 \pm 0.02) \times$ $10^{-6} \AA^{-2}$ and roughness of $23 \pm 4 \AA$ for both conditions. Throughout the experiment there is no difference between $\mathrm{H}_{2} \mathrm{O}$ vac and $\mathrm{D}_{2} \mathrm{O}$ vac which is consistent with all samples irrespective of their preparation parameters. This can be attributed to either no hydration water or strongly bound hydration water where the displacement of $\mathrm{H}_{2} \mathrm{O}$ by $\mathrm{D}_{2} \mathrm{O}$ molecules is suppressed. An isotopic exchange of $\mathrm{H}$ by $\mathrm{D}$ atoms in the polyelectrolytes can be excluded since both PSS and PDADMAC do not possess any displaceable acidic protons. In case of an exchange of hydration water in the dry multilayer, it is to be expected that the SLD obtained from the measurement against vacuum after exposure to $\mathrm{H}_{2} \mathrm{O}$ or $\mathrm{D}_{2} \mathrm{O}$ is different. The interpretation that no hydration water is exchanged in the course of varied environmental conditions is in agreement with the report of Ivanova et al.,${ }^{36}$ who stated that most of the $\mathrm{H}_{2} \mathrm{O}$ molecules found in polyelectrolyte multilayers at $0 \%$ relative humidity remain bound at $100 \%$ relative humidity $\mathrm{D}_{2} \mathrm{O}$.

\section{Effect of ionic strength}

Fig. 2 shows three different spectra of (PSS/PDADMAC) 6 prepared in aqueous $\mathrm{NaCl}$ solutions of various ionic strengths and against vacuum after exposure to $\mathrm{H}_{2} \mathrm{O}$. Upon adsorption of polyelectrolytes from solution of higher ionic strength the minima positions of the Kiessig fringes in the spectra shift towards low $Q$ and the distance, $\Delta Q$, between adjacent minima shrinks. The thicknesses derived from the fits for $0.1 \mathrm{M}$, $0.25 \mathrm{M}$ and $0.5 \mathrm{M}$ are $144 \pm 1 \AA, 267 \pm 6 \AA$, and $481 \pm 3 \AA$, respectively. The smearing out of the oscillations at high $Q$ is due to the roughness of the samples. The roughness obtained

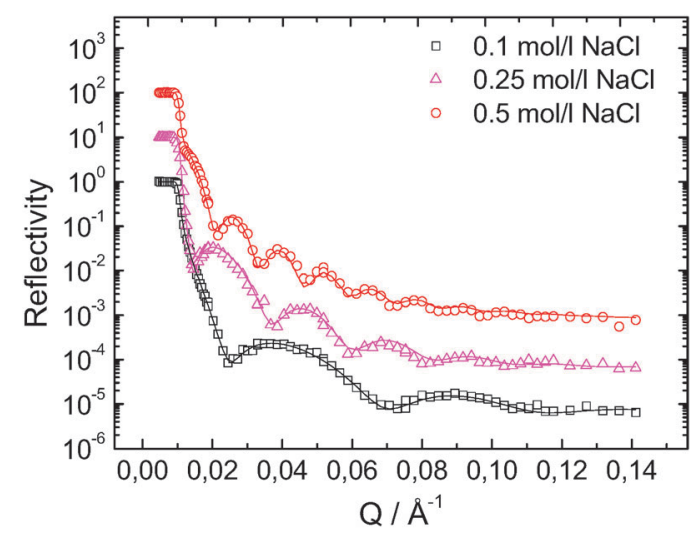

Fig. 2 Neutron reflectivity against vacuum after preparation $\left(\mathrm{H}_{2} \mathrm{O}\right.$ vac) showing the effect of ionic strength on multilayer thickness of (PSS/PDADMAC) 6 prepared from $0.1 \mathrm{M}, 0.25 \mathrm{M}$ and $0.5 \mathrm{M} \mathrm{NaCl}$ concentrations. The spectra of $0.25 \mathrm{M}$ and $0.5 \mathrm{M}$ have been multiplied by 10 and 100 , respectively, for clarity. 
for different ionic strengths is $10 \pm 4 \AA, 11 \pm 4 \AA$, and $22 \pm 5 \AA$ in order of increasing ionic strength. Hence, both multilayer thickness and roughness increase with increasing ionic strength. ${ }^{37,38}$ Addition of salt to the polyelectrolyte solution during multilayer preparation introduces counterions which also contribute to complex formation. ${ }^{39-41}$ By adsorbing polyelectrolyte from salt solutions of varying ionic strength, the layer thickness can be controlled over a wide range. Screening of the polyelectrolyte charges in a strong electrolyte solution leads to a smaller radius of gyration. Thus, adsorption of coils will take place, which occupy a lower surface area per chain, leading to a larger adsorbed amount of segments and consequently to a larger layer thickness. ${ }^{18,42}$ The pronounced difference in roughness between $0.25 \mathrm{M}$ and $0.5 \mathrm{M}$ is attributed to strong interdigitation of more coiled chains caused by higher ionic strength. The scattering length density does not show any systematic change with an increasing ionic strength.

\section{Effect of the type of ion}

The type of salt used during the multilayer preparation can affect the growth of the multilayer strongly. ${ }^{43,44}$ In order to investigate this effect (PSS/PDADMAC) 6 was prepared from $\mathrm{NaF}, \mathrm{NaCl}$ and $\mathrm{NaBr}$ solutions at a fixed ionic strength of $0.25 \mathrm{M}$. Different anions are chosen since anions are known to have a significantly larger effect on the thickness of the multilayers than their cation counterparts. ${ }^{45}$ Ion-specific effects become more important for cations above an ionic strength of $0.25 \mathrm{M}$ at which stage the influence is not negligible. The results of the neutron reflectivity measurements are shown in Fig. 3. At first sight, the distance, $\Delta Q$, between adjacent minima shrinks as the size of the anion of the respective salt increases. The position of the first minimum shifts towards low $Q$ values. From the fits the thickness obtained is $218 \pm 3 \AA, 267 \pm 6 \AA$ and $498 \pm 4 \AA$ for $\mathrm{NaF}, \mathrm{NaCl}$ and $\mathrm{NaBr}$, respectively. This shows an increase in the thickness of the multilayer as the size of the anion gets larger. The roughness obtained is $25 \pm 5 \AA, 11 \pm 4 \AA$ and $50 \pm 8 \AA$ for $\mathrm{NaF}, \mathrm{NaCl}$ and $\mathrm{NaBr}$, respectively. The roughness is expected to show a pattern similar to that of thickness, however, $\mathrm{NaF}$ is seen to produce exceptionally rougher multilayers. That explains why there is only one Kiessig oscillation and strong damping of $R$ at high $Q$ values. The effect of the anion on thickness and of the polyelectrolyte multilayers is due to its

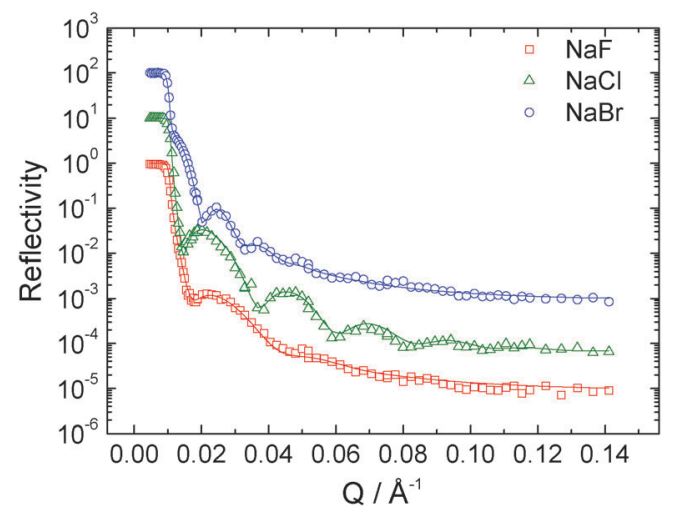

Fig. 3 Neutron reflectivity spectra of (PSS/PDADMAC) ${ }_{6}$ containing $0.25 \mathrm{M}$ concentration of $\mathrm{NaF}, \mathrm{NaCl}$ and $\mathrm{NaBr}$ against $\mathrm{H}_{2} \mathrm{O}$ vacuum. respective position in the Hofmeister Series ${ }^{46}$ and coincides with results of other studies on polyelectrolyte multilayers. ${ }^{23,24,40,45}$

\section{Quantitative analysis of water content}

A complete set of measurements as shown in Fig. 4, i.e., $\mathrm{H}_{2} \mathrm{O}$ vac, $\mathrm{H}_{2} \mathrm{O}$ liq, $\mathrm{D}_{2} \mathrm{O}$ liq and $\mathrm{D}_{2} \mathrm{O}$ vac, was performed for all $(\mathrm{PSS} / \mathrm{PDADMAC})_{6}$ multilayers which were prepared with different concentrations of $\mathrm{NaF}, \mathrm{NaCl}$ and $\mathrm{NaBr}$. In the following the quantitative analysis of the water content is described. The percentage of swelling in water is determined by:

$$
\phi_{\text {swell }}=\frac{d_{\text {swollen }}-d_{\text {dry }}}{d_{\text {swollen }}} \times 100
$$

where $\phi_{\text {swell }}$ is the percentage of swelling, $d_{\text {swollen }}$ is the thickness in the water swollen state and $d_{\text {dry }}$ is the thickness of the multilayer against vacuum. For example, the percentage of swelling in water of (PSS/PDADMAC) 6 prepared from aqueous solution of $0.1 \mathrm{M}, 0.25 \mathrm{M}$ and $0.5 \mathrm{M} \mathrm{NaCl}$ are $36 \%$, $43 \%$ and $55 \%$, respectively (see Fig. 5a). In many former studies the percentage of swelling was equated with the amount of water within the polyelectrolyte multilayer. However, it differs from the amount of water calculated by the concurrent change in the scattering length density of the multilayers. This leads to the conclusion that some hidden water exists which does not contribute to swelling in water but to the change in scattering length density only.

"Swelling" and "void water". Polyelectrolyte multilayers are deposited as layers that form continuous molecular layers without distinct layer-by-layer separation between polyelectrolytes of opposite charges, ${ }^{10-12}$ which in turn creates voids in the multilayer. In vacuum those voids within the multilayer are empty and the multilayer thickness is termed $d_{\text {dry }}$. Upon swelling the voids are filled with water called "void water". That water does not contribute to the swelling of the multilayers but exclusively to the change in scattering length density. Subsequent water absorbed by the multilayer, called "swelling water" on the opposite directly contributes to the swelling of the multilayer. The amount of "void water" $\phi_{\text {void, }}$, was retrieved from comparison of the amount of "swelling water", $\phi_{\text {swell }}$, calculated from eqn (1), with the total water

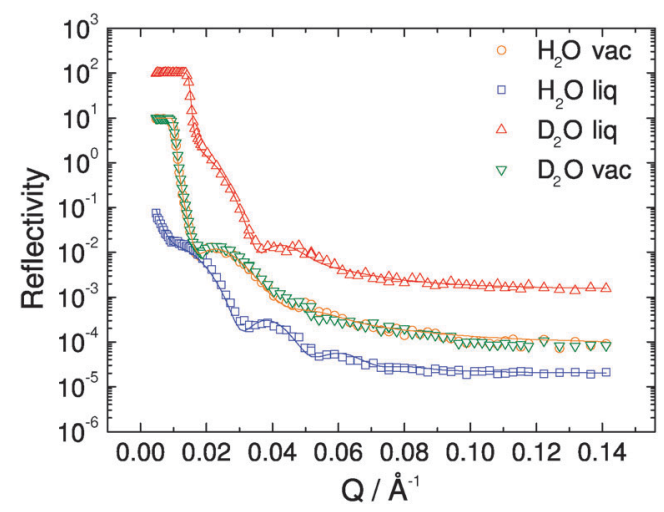

Fig. 4 Neutron reflectivity spectra of (PSS/PDADMAC) ${ }_{6}$ multilayers prepared from $0.25 \mathrm{M} \mathrm{NaF}$, showing the order of measurements. The reflectivities of the curves $\mathrm{H}_{2} \mathrm{O}$ vac, $\mathrm{D}_{2} \mathrm{O}$ vac and $\mathrm{D}_{2} \mathrm{O}$ liq have been multiplied by 10,10 and 100 , respectively. 

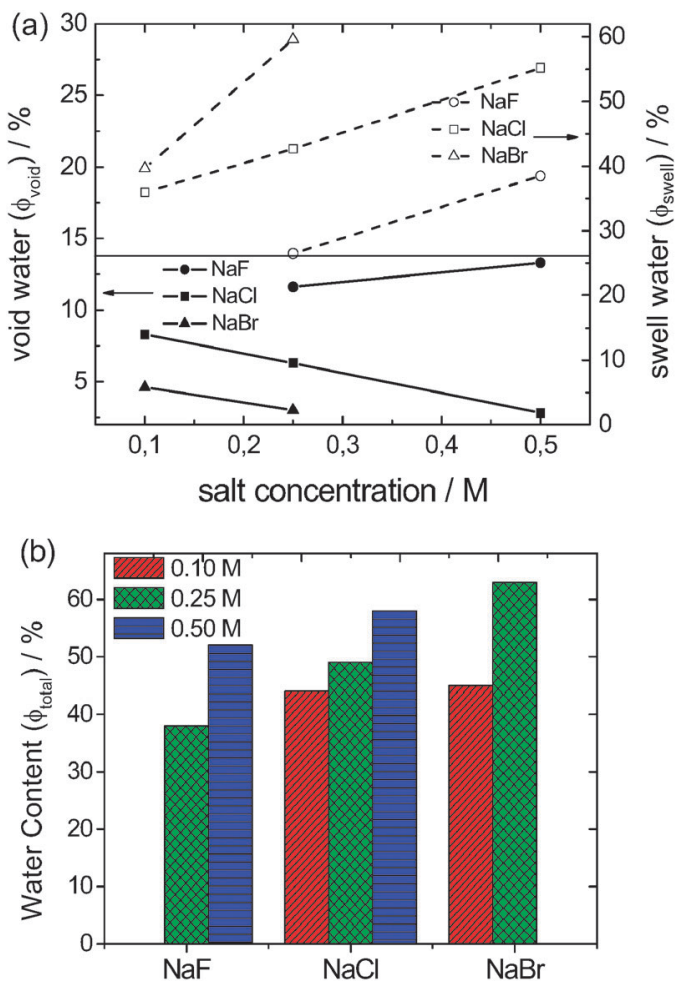

Fig. 5 (a) Amount of void water and swelling water in dependence of the salt concentration and type of ion of the preparation solutions. (b) Total amount of water in dependence of the salt concentration and type of ion of the preparation solutions.

content, $\phi_{\text {total }}$, calculated from the respective changes in the scattering length density and appropriate boundary conditions (see eqn (2)):

$$
\phi_{\text {total }}=(1-x)\left(1-\phi_{\text {swell }}\right)+\phi_{\text {swell }}=\phi_{\text {void }}+\phi_{\text {swell }}
$$

where $x$, the volume fraction of polymer, is given by

$$
x=\frac{N b_{\text {dry }}}{N b_{\text {water }}}-\frac{N b_{\text {swollen }}-\phi_{\text {swell }} N b_{\text {water }}}{\left(1-\phi_{\text {swell }}\right) N b_{\text {water }}}+1
$$

$N b_{\text {dry }}$ is the SLD of dry multilayer, $N b_{\text {swollen }}$ is the SLD of the swollen multilayer and $N b_{\text {water }}$ is the SLD of the water, $\mathrm{D}_{2} \mathrm{O}$ or $\mathrm{H}_{2} \mathrm{O}$. Hence, the total water content $\phi_{\text {total }}$ of a swollen multilayer is the sum of the "void water" $\phi_{\text {void }}$ and "swelling water" $\phi_{\text {swell }}$. The summary of the results for the amount of "void water" $\phi_{\text {void, }}$ "swelling water" $\phi_{\text {swell }}$ and total water $\phi_{\text {total }}$ is represented in Fig. 5. There is no significant difference in the water content for $\mathrm{H}_{2} \mathrm{O}$ and $\mathrm{D}_{2} \mathrm{O}$ in the multilayer. Therefore, the values presented in Fig. 5 are the average water content in the multilayers. We conclude that there is no isotopic effect in the thickness of the swollen multilayers as well as in the water content as can be seen from Table 1 and the ESI $\dagger$, respectively.

For each type of salt 3 different concentrations were used, i.e., $0.1 \mathrm{M}, 0.25 \mathrm{M}$ and $0.5 \mathrm{M}$. The results for $0.1 \mathrm{M} \mathrm{NaF}$ could not be provided because the multilayer was too thin to produce any oscillations and for $0.5 \mathrm{M} \mathrm{NaBr}$ the multilayer was unstable. With increasing ionic strength and increasing ion size the amount of total water and swelling water increases. The amount of void water shows an opposite effect: it decreases with increasing ionic strength (exception for $\mathrm{NaF}$ ). This means that the two water species (swelling and void water) partially compensate each other. In case of $0.1 \mathrm{M}$ the ion type has no effect on the total amount of water but on the amount of void and swelling water. Obviously, the increasing ion size has qualitatively the same effect as increasing the ionic strength.

\section{Discussion}

The main findings can be summarized as follows: (i) there is no displacement of hydration water in the dry polyelectrolyte multilayers and there is no replacement of $H$ by $D$. (ii) The thickness of the polyelectrolyte multilayers increases with increasing ionic strength of the polyelectrolyte solution. (iii) The thickness of the multilayers increases in accordance with the arrangement of the anions in the Hofmeister Series. (iv) The total water content of the polyelectrolyte multilayers increases with increasing ionic strength and also the type of ion. In order to qualitatively explain these findings, we will discuss the hydrophobic effect and the Hofmeister effect on polyelectrolyte multilayers.

\section{Hydration water in polyelectrolyte multilayers}

The generally accepted model of hydration ${ }^{47}$ invokes a cage of water molecules around a nonpolar solute molecule or molecular part, i.e. polymer backbone. Hydrogen bonds are preserved by partial ordering of water (a decrease in entropy), and contact with the solute can generate favorable, though small, enthalpies of dissolution. Hydrophobic interactions are generated when multiple nonpolar solutes, or fragments of molecules, associate to maximize contact of water with water, maintaining the structure and hydrogen bonds. Polyelectrolyte multilayers prepared by alternating adsorption of polyanions and polycations from aqueous solution are assumed to contain

Table 1 The structure of (PSS/PDADMAC) ${ }_{6}$ multilayer prepared from aqueous polyelectrolyte solution of $\mathrm{NaF}, \mathrm{NaCl}$ and $\mathrm{NaBr}$. Data set $\mathrm{H}_{2} \mathrm{O}$

\begin{tabular}{|c|c|c|c|c|c|c|c|c|}
\hline $\begin{array}{l}\text { Concentration of } \\
\text { salt }\end{array}$ & $\begin{array}{l}d\left(\mathrm{H}_{2} \mathrm{O}\right) d_{\text {dry }} \\
(\AA)\end{array}$ & $\begin{array}{l}d\left(\mathrm{H}_{2} \mathrm{O}\right) d_{\text {swollen }} \\
(\AA)\end{array}$ & $\begin{array}{l}d\left(\mathrm{D}_{2} \mathrm{O}\right) d_{\text {swollen }} \\
(\AA)\end{array}$ & $\begin{array}{l}\sigma\left(\mathrm{H}_{2} \mathrm{O}\right) \sigma_{\text {dry }} \\
(\AA)\end{array}$ & $\begin{array}{l}\sigma\left(\mathrm{H}_{2} \mathrm{O}\right) \sigma_{\text {swollen }} \\
(\AA)\end{array}$ & $\begin{array}{l}\mathrm{SLD}\left(\mathrm{H}_{2} \mathrm{O}\right) \\
\rho_{\mathrm{dry}}\left(10^{-6}\right. \\
\left.\AA^{-2}\right)\end{array}$ & $\begin{array}{l}\mathrm{SLD}\left(\mathrm{H}_{2} \mathrm{O}\right) \\
\rho_{\text {swollen }}\left(10^{-6}\right. \\
\left.\AA^{-2}\right)\end{array}$ & $\begin{array}{l}\operatorname{SLD}\left(\mathrm{D}_{2} \mathrm{O}\right) \\
\rho_{\text {swollen }}\left(10^{-6}\right. \\
\left.\AA^{-2}\right)\end{array}$ \\
\hline $0.25 \mathrm{M} \mathrm{NaF}$ & $218 \pm 3$ & $295 \pm 5$ & $295 \pm 6$ & $25 \pm 5$ & $40 \pm 6$ & $0.95 \pm 0.02$ & $0.53 \pm 0.03$ & $3.48 \pm 0.3$ \\
\hline $0.5 \mathrm{M} \mathrm{NaF}$ & $300 \pm 5$ & $486 \pm 8$ & $495 \pm 10$ & $34 \pm 6$ & $55 \pm 5$ & $1.20 \pm 0.03$ & $0.45 \pm 0.02$ & $4.04 \pm 0.2$ \\
\hline $0.1 \mathrm{M} \mathrm{NaCl}$ & $144 \pm 1$ & $226 \pm 3$ & $226 \pm 2$ & $10 \pm 4$ & $27 \pm 4$ & $1.33 \pm 0.02$ & $0.58 \pm 0.01$ & $3.47 \pm 0.1$ \\
\hline $0.25 \mathrm{M} \mathrm{NaCl}$ & $267 \pm 6$ & $465 \pm 9$ & $480 \pm 15$ & $11 \pm 4$ & $35 \pm 13$ & $1.34 \pm 0.03$ & $0.49 \pm 0.02$ & $4.00 \pm 0.2$ \\
\hline $0.5 \mathrm{M} \mathrm{NaCl}$ & $481 \pm 3$ & $1041 \pm 18$ & $1110 \pm 30$ & $22 \pm 5$ & $48 \pm 19$ & $0.74 \pm 0.04$ & $0.23 \pm 0.07$ & $4.11 \pm 0.1$ \\
\hline $0.1 \mathrm{M} \mathrm{NaBr}$ & $229 \pm 5$ & $395 \pm 6$ & $381 \pm 9$ & $23 \pm 4$ & $36 \pm 9$ & $1.04 \pm 0.02$ & $0.34 \pm 0.05$ & $3.45 \pm 0.2$ \\
\hline $0.25 \mathrm{M} \mathrm{NaBr}$ & $498 \pm 4$ & $1282 \pm 20$ & $1200 \pm 40$ & $50 \pm 8$ & $123 \pm 9$ & $1.05 \pm 0.02$ & $0.05 \pm 0.01$ & $4.36 \pm 0.1$ \\
\hline
\end{tabular}
vac and $\mathrm{D}_{2} \mathrm{O}$ vac are the same. Error bars are set in accordance to a level of $10 \%$ increase in $\chi^{2}$ 
hydration water in their dry state. Evidence was reported by Schwarz and Schoenhoff ${ }^{48}$ who applied ${ }^{1} \mathrm{H}$ NMR transverse relaxation to monitor the hydration water in polyelectrolyte multilayers. Schlenoff et al. ${ }^{49}$ also reported hydration contributions to association in polyelectrolyte multilayers. Our analysis based on neutron reflectivity indicates no structural change in the dry polyelectrolyte multilayers, prepared from light water $\mathrm{H}_{2} \mathrm{O}$, dried and subsequently exposed to heavy water $\mathrm{D}_{2} \mathrm{O}$ for 8 hours and then dried. This result was proven at different salt concentrations of $\mathrm{NaF}, \mathrm{NaCl}$ and $\mathrm{NaBr}$. For all samples the reflectivity spectra of $\mathrm{H}_{2} \mathrm{O}$ vac and $\mathrm{D}_{2} \mathrm{O}$ vac were identical producing the same multilayer thickness, same scattering length density and multilayer roughness. If hydration water exists in vacuum, then it is included in $x$, the volume fraction of polymer as fixed, not displaced (as integral part that cannot be resolved by the applied technique). The explanation for this phenomenon would be the presence of strongly bound hydration water surrounding the molecules of the polyelectrolytes making it impossible for the $\mathrm{H}$ in the hydrogen bonds to be displaced by $\mathrm{D}$, which is not fully understood so far. Similar effects are reported by Ivanova et al., who observed about 6 immobile protons at $0 \%$ r.h. in a PSS/PAH multilayer system. ${ }^{36}$ It could be imagined that by supplying the system with energy in the form of heat, more first-shell water conformations would be made accessible. Melting the cages of water molecules would then allow a closer approach of the polymer groups. The observed variations in SLD of the films in the dry state are caused by two effects: (i) variations in polymer volume fraction, $x$, and (ii) amount of bound water included in $x$. While with our technique we can resolve $x$, we cannot resolve the amount of bound hydration water associated to the polyelectrolyte with certainty. The upper limit for the SLD of the polymer complex itself without hydration water is $1.43 \times 10^{-6} \AA^{-2}$. This number is based on published data on the molecular volumes of PSS and PDADMAC of $201 \AA^{317}$ and $156 \AA^{3}{ }^{29}$ respectively, the latter based on the measured mass density of PDADMAC of $1.19 \mathrm{~g}$ $\mathrm{cm}^{-3}$. From our measured SLD values against vacuum we have to conclude that besides the polymer volume fraction also the amount of bound water varies as a function of preparation conditions.

\section{Effect of ionic strength and the type of ion}

The properties of the polyelectrolytes constituting the multilayer determine its structure and the charge compensation mechanism. The presence of salt in the solution can create electrostatic screening of the charge on the polyelectrolyte layer and also influence both the dynamics of polyanion/ polycation complexes holding the layers together as well as the forces operating in the system. ${ }^{50-52}$ Polyelectrolyte multilayers prepared from $\mathrm{NaBr}$ salt were thicker than those prepared from $\mathrm{NaF}$ due to the effect of the anions and their position in the Hofmeister Series. ${ }^{23,24,40,45,46}$ The Hofmeister Series usually presents an inversion point ${ }^{45,46}$ at about $\mathrm{Cl}^{-}$(for anions) and $\mathrm{Na}^{+}$(for cations). Hence, the position of these two ions is usually considered as a null point in the ion-specific effects. This explains why the $\mathrm{NaCl}$ multilayer has the smallest roughness in Fig. 3 and Table 1. In contrast to the thickness the roughness does not follow systematically the Hofmeister Series. The increasing coiling of the chains leading to an increasing film thickness has two counteracting effects on the roughness. A slight increasing flexibility of the PDADMAC chains due to a stronger interaction with anions from $\mathrm{F}^{-}$to $\mathrm{Cl}^{-}$leads to a stronger ability for rearrangement and therefore to a smoother interface. A further increasing coiling (from $\mathrm{Cl}^{-}$ to $\mathrm{Br}^{-}$) leads to rougher surfaces. ${ }^{40,45,53}$ The thickness of the multilayers prepared using different sodium salts $(\mathrm{NaF}, \mathrm{NaCl}$, $\mathrm{NaBr}$ ) at an ionic strength of $0.25 \mathrm{M}$ increased in the order of $\mathrm{F}^{-}<\mathrm{Cl}^{-}<\mathrm{Br}^{-}{ }^{24,54}$ The roughness increases in the same order with increasing ionic strength due to an increase in coiling. The scattering length density of the dry multilayer varies from $(0.95 \pm 0.02) \times 10^{-6} \AA^{-2}$ in the case of $\mathrm{NaF}$ to $(1.34 \pm 0.02) \times 10^{-6} \AA^{-2}$ in the case of $\mathrm{NaCl}$, with $\mathrm{NaBr}$ having $(1.05 \pm 0.02) \times 10^{-6} \AA^{-2}$. An opposite effect between roughness and SLD is observed among the multilayers of $\mathrm{NaF}, \mathrm{NaCl}$ and $\mathrm{NaBr}$. Whereas the multilayer of $0.25 \mathrm{M}$ $\mathrm{NaCl}$ shows lowest roughness, it also has the highest SLD among the multilayers of the sodium salts.

At ionic strengths up to $0.1 \mathrm{M}$ the counterions involved in extrinsic complex formation with the polyions are few. This increases electrostatic attraction between oppositely charged layers and causes the polyelectrolyte chains to conform flat to the substrate. In this case the layers are tightly bound to each other resulting in low multilayer thickness, roughness and voids. This accounts for the fact that multilayers prepared from $0.1 \mathrm{M} \mathrm{NaCl}$ and $0.1 \mathrm{M} \mathrm{NaBr}$ have the same total water content of $44 \%$ and $45 \%$, respectively. Therefore at low ionic strength the electrostatic interactions are dominating and specific ion effects are minor as shown in the present study. Above an ionic strength of $0.1 \mathrm{M}$ salt specific ion effects come into play. This observation supports findings of former studies. ${ }^{45}$ It has been shown that at high ionic strength (above $0.1 \mathrm{M}$ ), where the electrostatic interactions are partially screened, the interion interactions are controlled by dispersion forces that depend on the polarizability of the ionic species. ${ }^{55}$

\section{Swelling behavior and water content of polyelectrolyte multilayers}

The results show that the amount of void water and swelling water shows opposite dependencies on the ionic strength and the ion size leading to a partial compensation with respect to the total amount of water within the polyelectrolyte multilayers. Starting with the swelling water, the polyelectrolyte multilayer can be considered as a sponge. Due to stronger interaction of the PDADMAC with $\mathrm{Br}^{-}$more loops are formed, which are less fixed by oppositely charged polyelectrolytes than in the presence of $\mathrm{F}^{-}$during preparation. These loops will be folded during drying and have a strong ability for unfolding, i.e. reswelling, in the presence of water. The same effect occurs at high ionic strength.

In contrast, the amount of void water, which does not contribute to the swelling, rather probes the structure of the dry state. Less void water means a denser structure in the dry state. A higher flexibility of the chains leads to an easier reorganisation of the chains and therefore a denser packing. A higher flexibility is reached by intrachain screening caused 
by a high ionic strength and/or large counter ions. With increasing salt concentration and increasing size of ion, the total amount of water increases which indicates a decrease of polyelectrolyte density in the swollen state. This contradicts a former experiment on rhodamine transport through polyelectrolyte multilayers. An explanation might be that rhodamine also probes defects in the film. ${ }^{20}$

\section{Conclusions}

In this study, the influence of ionic strength and the type of ion (during preparation) on the water content of polyelectrolyte multilayers has been investigated by neutron reflectivity. It is found that there is no exchange of hydration water or replacement of $\mathrm{H}$ by $\mathrm{D}$ when dry polyelectrolyte multilayers prepared from $\mathrm{H}_{2} \mathrm{O}$, are incubated in $\mathrm{D}_{2} \mathrm{O}$ liquid for at least eight hours.

The polyelectrolyte multilayer acts like a sponge. The total water content of the polyelectrolyte multilayer is the sum of the "void water" which fills the empty space (excluded volume) between the polyelectrolyte chains and does not contribute to the swelling but to the change in scattering length density and the "swelling water" which contributes directly to swelling. "Swelling water" and total water content of the multilayer increase with increasing ionic strength and increasing size of the anions. With the increasing ionic strength of the polyelectrolyte solutions the amount of counterions in the aqueous medium contributing to the extrinsic polyelectrolyte complex also increase, resulting in screening of electrostatic attraction between oppositely charged polyelectrolyte segments. Increasing screening effect causes the polyelectrolyte chains to change from flat conformation to coil conformation thereby increasing the total thickness of the multilayer and the roughness.

In contrast, the amount of void water shows the opposite effect. It decreases with increasing ionic strength and anion size reflecting a denser structure in the dry state at high ionic strength and for large anions.

In the experiments shown increasing anion size acts like increasing the salt concentration. The specific ion effects become pronounced at an ionic strength larger than $0.1 \mathrm{M}$, where the electrostatics are mainly screened and dispersion forces between the ions become dominant.

\section{Acknowledgements}

We thank the Helmholtz-Zentrum Berlin für Materialien und Energie $\mathrm{GmbH}$ for its support in providing us with beam time for neutron reflectivity measurement at the V6 instrument. We also thank M. Kreuzer for helping during the experimental cycles. We appreciate the financial support by the Deutsche Forschungsgemeinschaft within the Priority Program SPP 1369 (K1 1165/11-1) and also financial support by the CoE "Unifying Concepts in Catalysis" (UniCat).

\section{References}

1 G. Decher and J. D. Hong, Makromol. Chem., Macromol. Symp., 1991, 46, 321.

2 G. Decher, J. D. Hong and J. Schmitt, Thin Solid Films, 1992, 210 , 831-835.

3 G. Decher, Science, 1997, 277, 1232.
4 P. Hammond, Curr. Opin. Colloid Interface Sci., 2000, 4, 430.

5 P. Bertrand, A. Jonas, A. Laschewsky and R. Legras, Macromol. Rapid Commun., 2000, 21, 319-348.

6 F. Caruso, Adv. Mater., 2001, 13, 11.

7 G. Decher, M. Eckle, J. Schmitt and B. Struth, Curr. Opin. Colloid Interface Sci., 1998, 3, 32-39.

8 R. Steitz, W. Jaeger and R. von Klitzing, Langmuir, 2001, 17, 4471-4474.

9 M. Gopinadhan, H. Ahrens, J. U. Gunther, R. Steitz and C. A. Helm, Macromolecules, 2005, 38, 5228-5235.

10 J. Schmitt, T. Grunewald, G. Decher, P. S. Pershan, K. Kjaer and M. Losche, Macromolecules, 1993, 26, 7058-7063.

11 M. Tarabia, H. Hong, D. Davidov, S. Kirstein, R. Steitz, R. Neumann and Y. Avny, J. Appl. Phys., 1998, 83, 725-732.

12 H. P. Hong, R. Steitz, S. Kirstein and D. Davidov, Adv. Mater., 1998, 10, 1104.

13 R. von Klitzing and H. Mohwald, Langmuir, 1995, 11, 3554-3559.

14 G. B. Sukhorukov, E. Dontah, H. Lichtenfeld, E. Knippel, M. Knippel, A. Budde and H. Möhwald, Colloids Surf., A: Physicochem. Eng. Aspects, 1998, 137, 253-266.

15 F. Caruso, D. Furlong, K. Ariga, I. Ichinose and T. Kunitake, Langmuir, 1998, 14, 4559-4565.

16 P. A. Neff, A. Naji, C. Ecker, B. Nickel, R. Von Klitzing and A. R. Bausch, Macromolecules, 2006, 39, 463-466.

17 M. Losche, J. Schmitt, G. Decher, W. G. Bouwman and K. Kjaer, Macromolecules, 1998, 31, 8893-8906.

18 H. A. Van der Schee and J. Lyklema, J. Phys. Chem., 1984, 88, 6661-6667.

19 M. R. Bohmer, O. A. Evers and J. M. Scheutjens, Macromolecules, 1990, 23, 2288-2301.

20 R. von Klitzing and H. Mohwald, Macromolecules, 1996, 29, 6901-6906.

21 G. Ahn-Ercan, H. Krienke and W. Kunz, Curr. Opin. Colloid Interface Sci., 2004, 9, 92-96.

22 V. Vlachy, B. Hribar-Lee, Y. V. Kalyuzhnyi and K. A. Dill, Curr. Opin. Colloid Interface Sci., 2004, 9, 92-96.

23 R. von Klitzing, J. E. Wong, W. Jaeger and R. Steitz, Curr. Opin. Colloid Interface Sci., 2004, 9, 158-162.

24 M. Salomäki, P. Tervasmäki, S. Areva and J. Kankare, Langmuir, 2004, 20, 3679-3683.

25 J. E. Wong, F. Rehfeldt, P. Hanni, M. Tanaka and R. von Klitzing, Macromolecules, 2004, 37, 7285-7289.

26 M. D. Miller and M. L. Bruening, Chem. Mater., 2005, 17, 5375-5381.

27 R. Steitz, V. Leiner, R. Siebrecht and R. von Klitzing, Colloids Surf., A: Physicochem. Eng. Aspects, 2000, 163, 63-70.

28 M. Gopinadhan, O. Ivanova, H. Ahrens, J. Guenther, R. Steitz and C. A. Helm, J. Phys. Chem. B, 2007, 111, 8426-8434.

29 H. Dautzenberg, E. Gornitz and W. Jaeger, Macromol. Chem. Phys., 1998, 199, 1561-1571.

30 D. Ruppelt, J. Kotz, W. Jaeger, S. E. Friberg and R. A. Mackay, Langmuir, 1997, 13, 3316-3319.

31 V. Bosio, G. F. A. Dubreuil and F. Bogdanovic, Colloids Surf., A: Physicochem. Eng. Aspects, 2004, 243, 147-155.

32 S. K. Satija, C. F. Majkrzak, T. P. Russell, S. K. Sinha, E. B. Sirota and G. J. Hughes, Macromolecules, 1990, 23, 3860-3864.

33 J. R. Howse, E. Manzanares-Papayanopoulos, I. A. McLure, J. Bowers, R. Steitz and G. H. Findenegg, J. Chem. Phys., 2002, 116, 7177-7188.

34 F. Mezei, R. Golub, F. Klose and H. Toews, Phys. B: Condens. Matter Quanta, 1995, 213, 898-900.

35 L. G. Parratt, Phys. Rev., 1954, 95, 359-369.

36 O. Ivanova, O. Soltwedel, M. Gopinadhan, R. Koehler, R. Steitz and C. A. Helm, Macromolecules, 2008, 41, 7179-7185.

37 U. Voigt, W. Jaeger, G. H. Findenegg and R. von Klitzing, J. Phys. Chem. B, 2003, 107, 5273-5280.

38 U. Voigt, V. Khrenov, K. Thuer, M. Hahn, W. Jaeger and R. von Klitzing, J. Phys.: Condens. Matter, 2003, 15, S213-S218.

39 S. Bharadwaj, R. Montazeri and D. T. Haynie, Langmuir, 2006, 22, 6093-6101.

40 R. von Klitzing, Phys. Chem. Chem. Phys., 2006, 8, 5012-5033.

41 P. Nazaran, V. Bosio, W. Jaeger, D. F. Anghel and v. R. Klitzing, J. Phys. Chem. B, 2007, 111, 8572-8581.

42 M. Schoenhoff, J. Phys.: Condens. Matter, 2003, 15, R1781-R1808. 
43 H. Mjahed, J. Voegel, B. Senger, A. Chassepot, A. Rameau, V. Ball, P. Schaaf and F. Boulmedais, Soft Matter, 2009, 5, 2269-2276.

44 J. Ruths, F. Essler, G. Decher and H. Riegler, Langmuir, 2000, 16, 8871-8878.

45 J. E. Wong, H. Zastrow, W. Jaeger and R. von Klitzing, Langmuir, 2009, 25, 14061-14070.

46 F. Hofmeister, Arch. Exp. Pathol. Pharmakol., 1888, 24, 247-260.

47 V. Yaminsky and E. Vogler, Curr. Opin. Colloid Interface Sci., 2001, 6, 342-349.

48 B. Schwarz and M. Schoenhoff, Colloids Surf., A: Physicochem. Eng. Aspects, 2002, 198, 293-304.
49 J. B. Schlenoff, A. H. Rmaile and C. B. Bucur, J. Am. Chem. Soc., 2008, 130, 13589-13597.

50 N. A. Peppas and A. R. Khare, Adv. Drug Delivery Rev., 1993, 11, $1-35$.

51 S. T. Dubas and J. B. Schlenoff, Langmuir, 2001, 17, 7725-7727.

52 G. B. Sukhorukov, J. Schmitt and G. Decher, Phys. Chem. Chem. Phys., 1996, 100, 948-953.

53 J. H. Bongaerts, J. J. Cooper-White and J. R. Stokes, Biomacromolecules, 2009, 10, 1287-1294.

54 M. Salomäki, T. Laiho and J. Kankare, Macromolecules, 2004, 37, 9585-9590.

55 B. W. Ninham and V. Yaminsky, Langmuir, 1997, 13, 2097-2108. 